\title{
APRENDIZAJES ACUMULATIVO Y GENERATIVO DE LAS ORGANIZACIONES EDUCATIVAS
}

\author{
José Cardona Andújar \\ Profesor de la Facultad de Educación, UNED \\ Madrid, España
}

Recibido 15-IV-2008 • Aceptado13-V-2008 • Corregido 21-VI-2008

\begin{abstract}
Resumen: En el presente ensayo se construye conocimiento, en primer lugar, acerca del concepto de las organizaciones en general, y de la escuela como organización específica. La escuela y otras instituciones educativas pueden considerarse como seres vivos $y$, desde esta premisa, son susceptibles de transformación y sujetas a un aprendizaje permanente. No obstante lo anterior, se profundiza en las características que presentan aquéllas que, verdaderamente, realizan un auténtico cambio hacia la mejora de la formación de su alumnado y al desarrollo profesional de sus docentes.

En un segundo momento, el autor aborda el tema del aprendizaje organizativo de las instituciones educativas, analizando las diferencias que presentan los aprendizajes acumulativo y generativo, defiende la complementariedad entre ambos aprendizajes, subrayando la necesidad de buscar la dimensión científica del conocimiento organizativo. Finaliza el trabajo abordando la problemática de los denominados facilitadores del aprendizaje de las instituciones educativas, asi como un conjunto de indicadores de este aprendizaje. En este sentido, unos y otros constituyen verdaderos y valiosos criterios en los que fundamentar el desarrollo de los procesos de evaluación y de acreditación de los centros educativos.
\end{abstract}

Palabras clave: Organización, escuela, aprendizaje de las organizaciones, organizaciones saludables, habilidad, capacidad, competencia, innovación, aprendizaje acumulativo, aprendizaje generativo, conocimiento técnico, conocimiento científico, factores, indicadores.

\section{Las organizaciones educativas}

Las organizaciones en general, y las educativas en particular, son seres vivos que nacen y crecen, se desarrollan a lo largo de su existencia; son seres vivos que, como cualquier organismo animado, constituyen sistemas compuestos de diversos elementos que interactúan entre sí y con el medio que les rodea, o entorno, en un sentido evolutivo que las hace susceptibles de transformarse mediante sucesivos cambios. De manera que su definición no está exenta de cierta complejidad, por lo que, a veces, se recurre a lo metafórico para profundizar y clarificar su naturaleza.

Asumimos, y aplaudimos, las opiniones de aquellos colegas que establecen lazos analógicos entre las organizaciones y las máquinas y organismos (Morgan, 1990 citado en Martín-Moreno, Q., 1996); Lorenzo, 1994), desde los que fundamentan la naturaleza sistémica de las mismas, considerando, por una lógica elemental, que cualquier modificación efectuada en uno de sus órganos repercutirá en todos los demás modificando, en ocasiones intensamente, los parámetros básicos de su funcionamiento o actividad y, por ende, los 


\begin{abstract}
The article analyzes the concept of the organizations in general and the school like specific organization is reviewed. The educative school and other institutions can be considered as living beings and, from this premise, they are susceptible of transformation and they are subject to a permanent learning. Also, the subject of the organizational learning of the educative institutions is touched, analyzing the differences that present the learning of cumulative and generative type and the complementariness between both is defended, emphasizing the necessity of a scientific vision of the organizational knowledge. Finally, the problem of the so-called "learning facilitator" in the educative institutions works, as well as a set of indicators of this learning. In this sense, they establish criteria to base the development of the processes of evaluation and accreditation of the educative institutions.
\end{abstract}

Key words: Organization, school, healthful learning of the organizations, organizations, ability, capacity, competition, innovation, cumulative learning, generative learning, technical knowledge, scientific knowledge, factors, indicators. resultados que se derivan de los mismos. En lo que se refiere a las organizaciones educativas, nos parece acertada la metáfora del hospital, en tanto que la atención educativa individualizada a cada educando se corresponde con el tratamiento de aquél a cada uno de sus pacientes.

Desde su propia etimología (bien del latín ordinatio, dispositio, esto es, obrar o disponer; bien del griego órgano o instrumento para obrar), el término organización hace referencia a disponer, obrar o relacionar, entre otras acepciones, acciones, que le convienen a su concepto. Fundamentándonos en estas aportaciones de lo lingüístico, validadas y complementadas desde la observación de la realidad, podemos decir que una organización es aquella entidad resultante de la adecuada combinación de una serie de medios humanos, funcionales y materiales disponibles y que, en el marco de unas relaciones determinadas, se orienta a la consecución de unos objetivos.

Si el concepto matriz a que nos referimos en el párrafo anterior, lo trasladamos a la escuela como organización educativa, y aunque sea desde una perspectiva formal, la entenderemos como una organización compleja, con una serie de roles desempeñados mediante la interacción de las normas establecidas y en función de la propia personalidad de quienes los ejercen, y todo ello en el marco de unos objetivos que debe cumplir y de otras variables implicadas (Cardona, 2001). También se han referido a ella, concibiéndola como una microsociedad, con su cultura propia y su clima particular, a su vez integrados en múltiples subculturas que influyen en la conducta $\mathrm{y}$ actividad de sus miembros (Backman y Secord, 1968).

\section{Las organizaciones que aprenden}

Si bien, por su naturaleza, las organizaciones son perfectibles mediante un aprendizaje organizativo, unas consiguen 
mejorar actualizando sus potencialidades y otras no, por lo que parece razonable pensar que haya una serie de peculiaridades que diferencien las primeras de la segundas. Refiriéndose a los centros educativos como organizaciones, se entiende que en la mejora de una escuela influye significativamente el ritmo y la eficacia del cambio que protagonicen (Hopkins, 1996), dando lugar a la siguiente tipología de centros (en López y Sánchez, 2004):

Estancados: son aquellos centros en los que existe una percepción o sentimiento de fracaso, en los que cada aula es un compartimento estanco, aislada de las demás, y donde es difícil emprender procesos de cambio.

Paseantes: muy tradicionales, en los que sus profesores gozan de estabilidad y se mantienen de los éxitos pasados, siendo reticentes a nuevos cambios por considerar que la actual situación o funcionamiento de la institución es la adecuada.

Desencaminados: son los que se esfuerzan en una dirección de cambio aunque en demasiados ámbitos, por lo que su actividad no consigue los frutos deseados; adolecen de falta de claridad en sus objetivos y metas, y, sobre todo, en la prioridad de cada una de ellas, estando necesitados de una adecuada orientación y precisan de un asesoramiento eficaz.

Dinámicos: han conseguido la deseable armonía entre estabilidad y cambio, tienen claras sus metas y disponen de los recursos suficientes para abordarlas; apoyan su actividad en el trabajo en equipo o colaborativo, y fundamentan sus líneas de mejora en procesos sistemáticos de autoevaluación. Son estos centros lo que están en condiciones adecuadas para conseguir un aprendizaje organizativo, disponiendo de la cultura adecuada para ser organizaciones educativas que aprenden.

\subsection{Concepto y características de las organizaciones que aprenden}

Son aquellas que, asumiendo su naturaleza de organismos vivos, y dinámicos, son capaces de cambiar desde su responsabilidad de dar respuestas adecuadas a las demandas, siempre cambiantes, de una sociedad en constante transformación. Lo anterior implica potenciar su crecimiento, pero entendido éste como un ejercicio constante de auto-revisión que sea capaz de diagnosticar aquellos aspectos susceptibles de mejora y su priorización, lo cual constituye ya un verdadero aprendizaje, y que les va a exigir la profundización en las relaciones tanto internas como externas.

Aquella organización que aprende ha de presentar, a nuestro juicio, las siguientes características: a) Capacidad para diagnosticar situaciones o líneas de funcionamiento susceptibles de mejora, b) Abordan sistemáticamente la solución de sus problemas, b) Persiguen la búsqueda constante y metódica, desde el estilo más apropiado, de nuevo conocimiento organizativo (hablaremos de ello), c) Son capaces de consolidar su propio aprendizaje, y d) Están en buena disposición para transferir, a corto plazo, el saber adquirido a nuevas situaciones. $\mathrm{Y}$ es que, una organización que aprende intenta aprovechar su capacidad de reflexión crítica sobre la práctica, buscando espacios de análisis de los procesos, y transformar todo ello en fuente de su propio desarrollo (Díaz, 2000).

Para que todo lo anterior sea posible en el seno de las organizaciones educativas, convenimos que éstas han de ser saludables (Martín, 2003), siempre que por ello entendamos que han de promover activamente la autoestima de cada alumno, favorecer las buenas relaciones entre profesores y alumnos, facilitar vías para la información y la participación, y fomentar lazos sólidos entre centro, familias y comunidad en general. 


\subsection{El aprendizaje organizativo y su tipología}

Como sucede con todo constructo que se precie, son plurales las definiciones que se han construido para clarificar el concepto subyacente en esta expresión. Desde nuestra propia experiencia, consideramos que sobre aprendizaje organizativo, en tanto que producto, se podría hacer referencia mediante dos sustantivos: habilidad y capacidad (Ver mapa 1). Desde el primero de ellos, se consideraría como aquella habilidad de una organización para comprenderse a sí misma desde su propia experiencia, lo que no está nada mal si no fuera porque con el término habilidad se hace una clara connotación a destreza especialmente manual, lo que equivaldría a que la auto-compresión aludida estaría originada por una práctica carente del necesario fundamento teórico.

\section{Mapa 1}

Tipos de aprendizaje organizativo e innovación

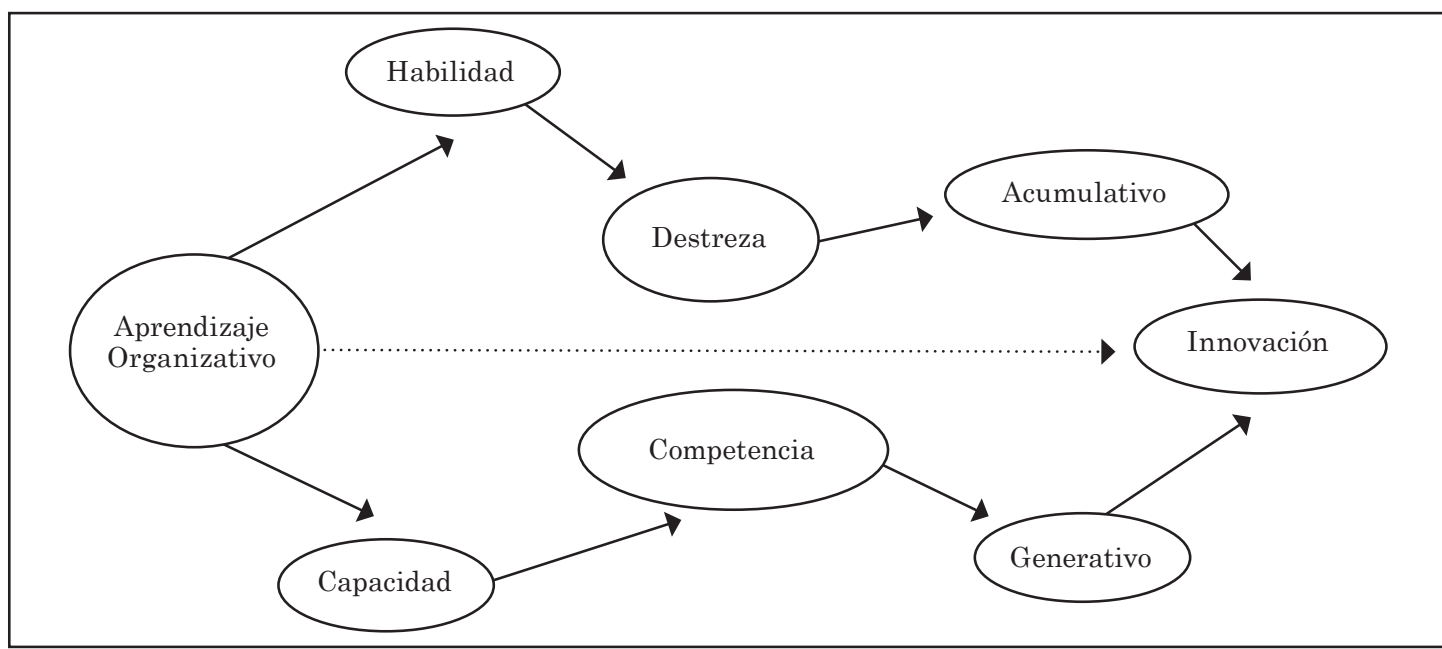

Bolívar parece asumir el aprendizaje organizativo desde el primer sustantivo, ya que afirma que con esta expresión nos referimos "a la habilidad de la gente, grupos y organizaciones para modificar los modos en que habitualmente piensan sobre cómo tratar y resolver los problemas" (Bolívar, 2000, p. 33). Ello es legítimo, pero entonces, y a nuestro juicio, estaríamos muy cercanos a ese tipo de aprendizaje denominado acumulativo (Senge, 1992), que ayuda a resolver los problemas en función de las formas que se han empleado habitualmente, por lo que, desde él, se limita una forma de proceder más imaginativa o creadora.

Por el contrario, el término capacidad establece una sinonimia más profunda con talento, competencia, aptitud o cualificación, que indican conceptos más nobles, muy cercanos a inteligencia, más próximos a lo que se denomina pensamiento divergente; de tal forma, que se ha considerado que aprendizaje organizativo es la capacidad para mantener o mejorar sus acciones, basado en la experiencia (Nevis, Dibella y Gould, 1995). En este sentido, y bajo nuestro punto de vista, estaríamos con más propiedad en lo que se ha llamado (Senge, o.c.) aprendizaje generativo, desde el que la resolución de los problemas que se presentan a una organización pasa por el reconocimiento de las fuentes sistémicas que los engendran (en Bolívar, o.c., p. 30).

Como se refleja en el Mapa 1 anterior, la adquisición de destrezas (aprendizaje acumulativo) conduce per se a un 
conjunto de habilidades profesionales (en el docente) e institucionales (en la organización educativa) estáticas, que no hacen sino reproducir aquellas competencias incluidas, respectivamente, bien en la formación inicial, bien en la propia norma o legislación educativa. Por el contrario, la capacidad demanda una actividad intelectual de mayor nivel y produce aprendizaje generativo, que capacita a profesores e instituciones educativas para dar una respuesta más flexible y creativa a las diversas y complejas situaciones de una sociedad que, como la actual, presenta un constante y acelerado cambio. A pesar de todo ello, innovar en educación exige a las instituciones docentes y a sus profesionales servirse de ambos tipos de conocimiento, pues en modo alguno han de considerarse antagónicos.

Para una conceptualización más rigurosa, habrá de tenerse en cuenta que el aprendizaje de las organizaciones implica un necesario, pero insuficiente, aprendizaje individual; habrá que trascender este último en el marco de iniciativas más globales y de procesos en colaboración, donde se produzca una integración de experiencias y saberes orientados a la mejora de los centros como organizaciones, sin olvidar que estos conforman, junto con sus entornos, comunidades educativas. Es en un adecuado conocimiento comunitario donde se produce el aprendizaje de estas instituciones, llamado también, como queda dicho, aprendizaje generativo.

Defendemos aquí que ambos tipos de aprendizaje organizacional, acumulativo y generativo, antes que mutuamente excluyentes, han de ser complementarios, de manera que contribuyan a la construcción de saberes que transformen las escuelas, ahora bien, teniendo en cuenta que sólo desde el segundo de ellos se afloran nuevos modelos mentales compartidos, expresados en una visión que favorezca tanto la colaboración como la comunicación, al tiempo que facilitan la transformación mental de sus elementos personales.

\section{Estilos de aprendizaje organizativo}

$\mathrm{Al}$ igual que cada estudiante tiene su propio estilo de aprender (lo que, en justa correspondencia, conlleva distintos estilos de enseñar), las organizaciones, de todo tipo (y, por tanto, también las educativas o centros docentes), también adoptan sus propios itinerarios o estilos de aprendizaje organizativo. En este sentido, y a nuestra forma de ver este fenómeno, habría que considerar dos estilos claramente diferenciados de aprendizaje organizativo:

\subsection{El AO técnico-práctico}

Extrapolando aquí nuestra reflexión epistemológica acerca de la naturaleza de la Organización Escolar como disciplina pedagógica (Cardona, 2001), diremos que es aquel aprendizaje que produce saber experiencial, esto es, fundamentado en la práctica organizativa y que, al elaborarse mediante una serie de acciones repetitivas, carece, en estricto sentido, de carácter científico; desde estos presupuestos, constituye un aprendizaje muy cercano a lo meramente técnico (en lo que éste tiene de conjunto de procedimientos y recursos que nos permiten dominar los fenómenos sin necesidad de comprender sus causas).

De forma que este aprendizaje organizativo práctico, así conceptuado, se caracteriza por una estructuración permanente, fruto de una actividad muy normativa y escasamente reflexiva. En todo caso, este estilo de aprendizaje, en nuestra opinión, cuestiona seriamente la innovación, en tanto que limita la consideración de variables reales que trasciendan la legislación del ámbito (o, simplemente, la interacción creativa de las que se contemplen en ella) y, sobre todo, aquel conocimiento que se genera en la realidad no formal de las organizaciones (muy rica en contenidos de aprendizaje sobre la cultura de las instituciones). 
Por otra parte, fiar el auto-conocimiento de las organizaciones a este estilo de aprender supone, en un primer momento, no reconocer la posibilidad de los elementos humanos de la organización para trascender la realidad, mejorándola desde la búsqueda del necesario principio de causalidad de los fenómenos; y en segundo lugar, este estilo de aprender adolece del olvido que registra de la dimensión dinámica y cambiante de la sociedad y de las organizaciones existentes en ella y, en consecuencia, la naturaleza de los problemas que en ellas se plantean. En definitiva, la naturaleza de este aprendizaje práctico o técnico produce como resultado la consecución de habilidades o hábitos, por lo que es insuficiente para enfrentar con éxito planteamientos creativos a los problemas emergentes de la dinámica organizacional.

\subsection{El AO tecnológico-científico}

Para profundizar en el concepto de este estilo de aprender, es necesario partir de unos planteamientos epistemológicos en los que militamos. En primer lugar, deberemos convenir en que lo técnico es precientífico, con un fundamento esencialmente empírico, mientras que con lo tecnológico nos situamos en terreno más experimental y científico, ya que un cuerpo de conocimiento es tecnológico si, y sólo si, es compatible con la ciencia coetánea y controlable con el método científico (Bunge, M., 1985).

En consecuencia con la premisa del párrafo anterior, consideramos que el estilo tecnológico o científico de aprendizaje organizativo es aquél basado en la explicación de la realidad de las organizaciones y cuyo objetivo esencial es transformarla buscando alternativas de mejora fundamentadas. De manera que, este estilo de aprendizaje, frente al técnico, supera el ámbito del hacer, incluso del hacer bien por mera experiencia, y nos conduce al saber por qué se hace, se aplica o realiza una determinada planificación de una forma y no de otra diferente. Esto es, este estilo de aprendizaje, por decirlo con palabras del profesor Gairín Sallán (Gairín, 1987, p. 139), “domina los fundamentos de la práctica, conoce otras formas de hacer y, por lo tanto, puede adaptar su acción a las nuevas exigencias que imponga las variables de la realidad".

Para finalizar este apartado, y por cuanto el quehacer organizativo en los centros docentes se orienta a la innovación optimizadora del contexto educativo, ha de basarse para ello en ambos estilos de aprendizaje, y no exclusivamente en el técnico.

\section{Factores que facilitan el Aprendizaje organizativo}

Con la denominación de facilitadores del aprendizaje en/de las organizaciones educativas hacemos referencia a patrones de funcionamiento de las mismas en el ámbito de la cultura institucional, así como de la planificación didáctico-organizativa y su correspondiente proceso de desarrollo. Este mismo significado le concede Bolívar (o.c., p. 182), cuando los considera como "el conjunto de estructuras y procesos que afectan a cómo de fácil o difícil es que ocurra el aprendizaje efectivo que tiene lugar en la realidad de los centros". Desde estas coordenadas, dichos facilitadores, que podrán ser de entrada y de proceso, tienen su referente en los paradigmas y teorías de organización que fundamenten este ámbito.

Nevis y colaboradores (1995) aportan una relación de indicadores favorecedores de aprendizaje organizativo, y que por su interés reproducimos tomados de Bolívar, o.c., p. 182). A saber: a) Exploración del entorno, b) Insatisfacción con las realizaciones actuales, c) Preocupación por la evaluación constante, d) Conjunto mental predispuesto a experimentar, e) Clima de apertura, f) Formación permanente de todos sus miembros, g) Variedad operativa en métodos, procedimientos y sistemas, h) Apuesta decidida por lo múltiple, i) Liderazgo 
participativo, y j) Clara interdependencia de las unidades organizativas, los problemas y sus soluciones.

Es evidente que la existencia, mejor aplicación, de los mencionados facilitadores requiere la implementación o puesta en marcha de una estrategia adecuada; estrategia que ha de apoyarse en un conjunto de principios, que, si bien habrá que contextualizar en cada caso, orientarán los procesos de mejora que se planifiquen institucionalmente con carácter general. En este sentido, nosotros (Cardona, 2000) proponemos una secuencia de intervención fundamentada en los siguientes principios:

La concienciación de los diferentes estamentos comunitarios hacia la reflexión colaborativa y el trabajo en equipo, como un estilo innovador y eficaz de planificación en las organizaciones docentes, que aglutine $\mathrm{y}$ sistematice, multiplicando de esta forma sus efectos, las diversas, y de otro modo inconexas, aportaciones individuales.

La optimización del conocimiento de los roles de cada miembro del centro, favoreciendo la autocrítica, definiendo funciones y responsabilidades $\mathrm{y}$ posibilitando la construcción de un organigrama adhocrático, que, trascendiéndolo, dote de la debida consistencia, racionalidad y eficiencia al organigrama prescriptivo y formal, facilitando, paralelamente, la posibilidad creadora del mismo (incorporando, así, a la organización en el ámbito del aprendizaje generativo).

La emergencia y consolidación de una cultura genuina, singularizada, en cada centro y su comunidad, estableciendo un quehacer dinámico y sistemático, permanente, que se proponga la mejora como finalidad irrenunciable.

La sensibilización de la comunidad educativa de cada centro hacia actuaciones de auto-revisión crítica (evaluación interna) para la innovación de su oferta formadora, facilitando la puesta en práctica de su proyecto institucional (educativo, curricular y organizativo), en tanto que parámetro fundamental de calidad.

El impulso sustantivador de la formación permanente y de la construcción de conocimiento profesional, vertebrados en torno a la reflexión sobre la práctica diaria, en tanto que procedimiento más idóneo para la innovación de la propia teoría didáctica y organizativa, así como fórmula valiosa para la elaboración de conocimiento profesional de los docentes y de su propia plenificación personal.

En este marco definido por los anteriores principios, cobra especial relevancia la dimensión autoevaluadora, toda vez que permite reflexionar sobre aquello que se hace (siempre pensando en asumir compromisos de mejora), facilita la función coordinadora (en tanto que propicia espacios para el trabajo en común de los profesores), impulsa el diálogo y la participación, suscita el aprendizaje de matices novedosos, permite tomar decisiones más racionales (corrigiendo errores), refuerza la coherencia de los equipos docentes, y ayuda, en suma, al perfeccionamiento de los profesores en su propio entorno (Santos, 1993).

Por su parte, Bolívar concibe los siguientes procesos clave en la configuración del centro docente como una organización que aprende (OA), considerando cuatro condiciones para fomentar dicha condición en la escuela; dos externos, cambios en el entorno, y en la política educativa, y dos internos, experiencia anterior de desarrollo, e historia y cultura escolar. Desde estos presupuestos citados, es necesario que el centro esté inmerso en programas integrados y compartidos de desarrollo, lo que presupone una aceptación compartida de visiones y necesidades que van provocando un cambio en la cultura escolar, que, al institucionalizarse, facilitan el desarrollo 
de la organización (Bolívar, o. c.). La interrelación de estas condiciones y programas puede ser mejor entendida en la siguiente Figura 1:

Figura 1

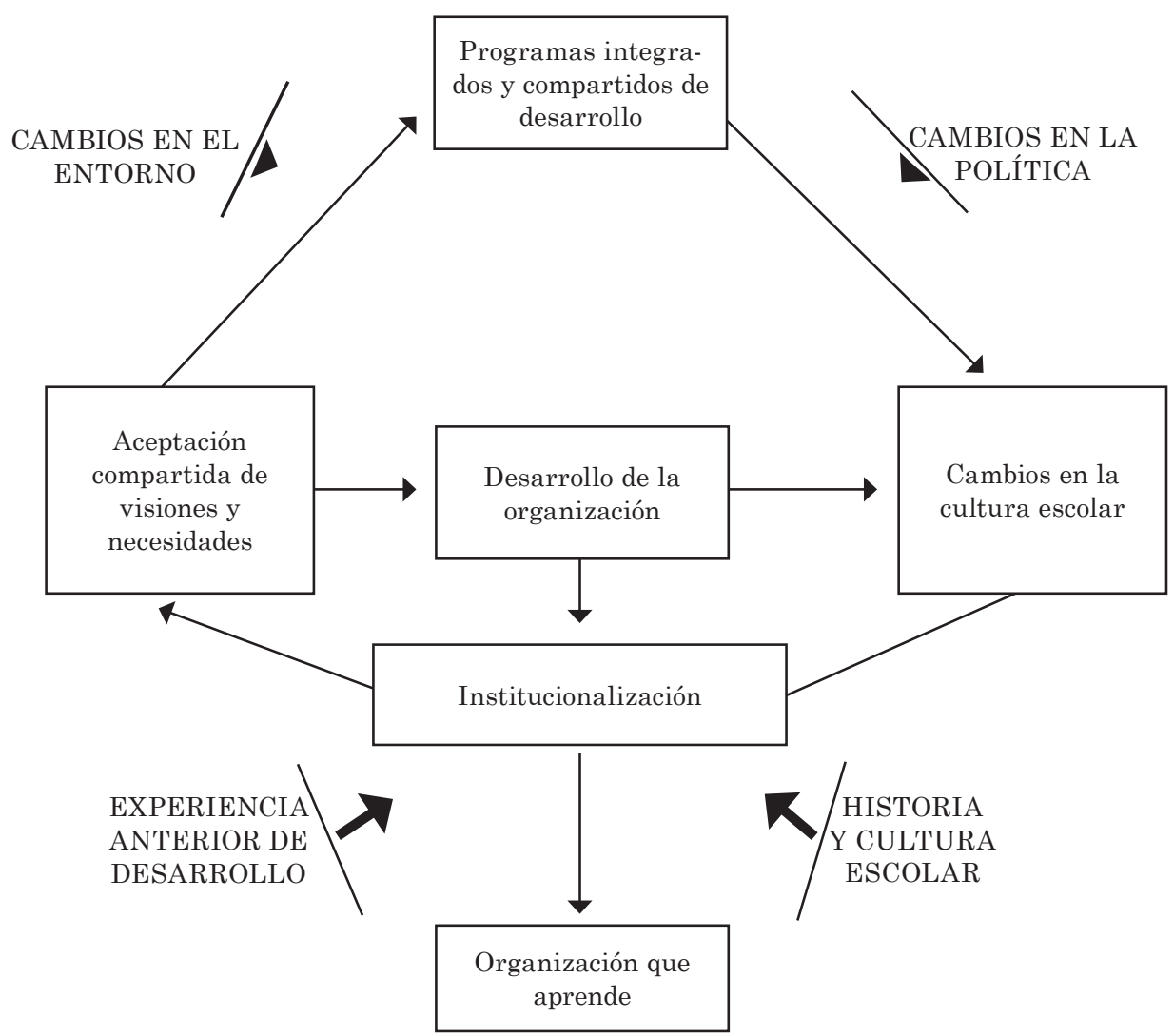

Figura 1: El centro educativo como organización que aprende (Bolívar, A., 2000, p. 193); adaptación.

Como podemos deducir fácilmente de la Figura 1, un adecuado aprendizaje de las organizaciones viene definido y explicado en función de dos tipos de macro-variables: las externas (que provienen del entorno, principalmente la política educativa: leyes, decretos, órdenes y/o resoluciones pertinentes), e internas (que tienen su origen en el intorno, significativamente influido por experiencias pretéritas y por la historia y cultura de la organización). Ambos escenarios descritos provocan transformaciones en la cultura escolar e influyen significativamente a la hora de diseñar, desarrollar y evaluar el propio currículo formativo de cada institución y el mismo sistema metodológico aplicado.

\section{Los indicadores del aprendizaje organizativo}

De la larga reflexión que precede, pueden deducirse las exigencias a plantear a la escuela que aprende (Learning Organization), y situándonos, como parece 
lógico, en el actual paradigma pluralista (comprensivo) que rige los nuevos enfoques de los centros docentes como organizaciones.

Debe considerarse que las instituciones educativas han de caracterizarse por su sensibilidad hacia las necesidades y demandas personales, profesionales, sociolaborales y axiológicas de sus agentes y clientes. No obstante, y con demasiada frecuencia, los fines de la escuela se focalizan con cierta exclusividad en los clientes (esto es, en los alumnos), olvidando que deben ser reivindicados y atendidos también aquellos objetivos que hacen de la organización escolar un espacio de construcción de conocimiento profesional y de realización personal de los docentes.

Invitamos, en consecuencia, a considerar los establecimientos escolares como instituciones críticas y renovadoras, como ecosistemas en los que la colaboración entre profesores, alumnos, familias y otros agentes de la comunidad escolar oriente un clima de comprensión y de diálogo democrático para la colaboración. Como decimos en otro trabajo:

"Es sólo en este clima donde puede hacerse realidad un desarrollo plenificador de los diferentes currículos y la construcción de conocimiento profesional de sus profesores; es en esta escuela donde va a ser posible la concienciación de los Claustros, Departamentos y Equipos ante las diferencias personales, sociales y familiares de los estudiantes" (Medina y Cardona, 1996).

Desde estos planteamientos, la escuela que aprende ha de superar aquellas actitudes de egocentrismo y aislamiento profesional, y sustituirlas por otras abiertas a reconocer las aportaciones de otros colegas y agentes comunitarios, en un clima que facilite el intercambio de experiencias, la asunción y superación de nuestros errores y una vivencia más intensa y enriquecedora de nuestra profesión de enseñantes.

En el sentido apuntado más arriba, la institución educativa capacitada para protagonizar un aprendizaje organizativo, deberá responder a un conjunto de señales o indicadores de calidad, esto es, ha de presentar un funcionamiento desde el que:

Asuma la singularidad de cada alumno y enseña en consecuencia: la pluralidad de culturas que, habitualmente, conviven en la mayoría de las aulas, invitan a caminar hacia una educación intercultural, en la que la existencia de grupos heterogéneos obliga a profundizar en el conocimiento de cada alumno para aplicarle el tratamiento educativo que, por su condición, necesita.

Promueva el desarrollo profesional de los profesores, entendiendo éste como un proceso pluridimensional de realización humana y profesional de los educadores, que ha de ser aceptado desde la auto-reflexión crítica y creativa, y, al tiempo, contextualizado en una determinada cultura, y orientado a la optimización de la tarea específica. Así percibido, incluiría un desarrollo centrado en las dimensiones pedagógica, cognitiva, teórica, profesional y, además, profundizando en el conocimiento y comprensión de sí mismo (Howey, 1985).

Potencie las relaciones con su comunidad educativa, auto-definiéndose como un elemento constitutivo de la misma, estimulando, paralelamente, la participación de sus diferentes estamentos, ya que entiende la comunidad escolar como un conjunto al que se incorporan, para una integración educadora, los diversos entornos educativos ubicados en su geografía, contribuyendo cada uno de ellos en función de la cultura que le es propia.

Fomente una metodología colaborativa, esto es, el trabajo en equipo de sus elementos o recursos humanos, entendiendo, y asumiendo, que la práctica colaborativa comprometida no es simplemente un término apropiado, ni siquiera un 
vocablo de excelente aceptación en un contexto social urgido de aportaciones solidarias, sino que es, esencialmente, un modo riguroso y posible de comprender las realidades educativas y de actuar en consecuencia (Medina, 1996).

Planifique y, en consecuencia, actúe desde las experiencias pre y extraescolares de su alumnado, fundamentando en ellas su labor educativa, estudiando el entorno como una de las bases y fundamentos de sus proyectos educativo y curricular, en un intento de evitar lo que se ha venido denominando discontinuidad horizontal y que tanto ha influido en el fracaso de ciertos programas formativos (la educación compensatoria en España, por citar sólo un ejemplo).

Diagnostique sistemáticamente lineas de mejora, fundamentando en ellas el cambio, a través de procesos de evaluación interna, que debe ser asumida como una actividad de autoreflexión colaborativa del equipo de colegas, o claustro, y que se proyecta en los diferentes ámbitos, áreas o variables del centro escolar.

Genere un clima social adecuado, que va a sintetizar el ecosistema de relaciones y el propio discurso, la cultura, los valores, las actitudes, así como las percepciones y las concepciones de cada persona, incidiendo todo ello en la interacción y toma de decisiones orientadas a transformar la institución en un marco de trabajo gratificante.

Se perciba como una comunidad profesional de aprendizaje, desde una visión del docente como un técnico que reflexiona sobre su práctica en el aula y centro, esto es, el profesor como práctico reflexivo que vive intensamente su escuela como objeto y lugar de conocimiento, concibiendo su profesionalización en una línea de colaboración con los demás miembros del equipo y orientada al trabajo en un proyecto común, en un proyecto asumido y consensuado de transformación e innovación del currículo y de él mismo como profesional (Cardona, 1998, p. 151). Y en consecuencia con ello, cree en la imposibilidad de un buen aprendizaje del alumnado sin un buen aprendizaje tanto de sus profesores como de ella misma como organización.

Asuma aprender de sus errores, considerando que lo más negativo del error es permanecer en él, perseverar en aquellas formas de pensar y actuar que han llevado a la institución a cometerlo. En esta línea, considera que un error es siempre un desafío para mejorar corrigiendo, una oportunidad valiosa para la retroalimentación, para el feed-back esperanzado en la perfectibilidad de las organizaciones y de las personas que en ella trabajan.

$\mathrm{Y}$, finalmente, que conciba la calidad educativa como un referente, conceptuándola como el ideal de perfección que se persigue, constituyéndose en la meta que orienta las acciones educativas. La escuela que aprende entiende, pues, la calidad como esa tensión utópica que mueve y motiva hacia la mejora permanente en todo cuanto se hace, y que, en clara armonía con ello, no tiene necesariamente que constituir un punto de llegada, sino, antes bien, una trayectoria o camino hacia lo mejor, un perfeccionamiento constante de todo cuanto hace en esa búsqueda, quizá inalcanzable, de la perfección humana.

Consideramos, ya para finalizar este trabajo, que las escuelas que tengan como guía de su actividad educadora los principios que se hacen operativos desde los anteriores indicadores, serán unas instituciones que aprenderán, constituyéndose, además, dichos principios en elementos definidores 
de un estilo de aprendizaje organizativo, aprendizaje en esa dimensión tecnológica y científica a que antes nos hemos referido, y que otros dan en llamar aprendizaje generativo o aprendizaje por exploración, y que son los más indicados para aquellas instituciones educativas que aspiren a colgarse el adjetivo de dinámicas.

\section{Referencias bibliograficas}

Backman, C. W. y Secord, P. F. (1996). A Social Psychological View of Education [Perspectiva socio-psicológica de la educación]. En Q. Martín-Moreno (Aut.). La Organización de Centros Educativos en una perspectiva de cambio (p. 89). Madrid: Sanz y Torres.

Bolívar, A. (2000). Los centros educativos como organizaciones que aprenden. Promesa y realidades. Madrid: La Muralla.

Bunge, M. (1985). Epistemología. Barcelona: Ariel.

Cardona, J. (1998). Cultura evaluativa de centros de educación. En S. Gento (Coord.), Gestión y supervisión de centros educativos (pp. 123-154). Buenos Aires: Docencia.

Cardona, J. (2000). Modelos de innovación educativa en la Educación Fisica. Madrid: UNED. (Colección: Educación Permanente).

Cardona, J. (2001). Elementos de teoría organizativa del centro escolar. Madrid: Sanz y Torres.

Díaz, T. (2000). Organizaciones que aprenden y su certificación en la universidad. En M. Lorenzo y otros (Coords.), Organización Escolar diferencial. Modelos y estrategias (pp. 1637-1644). Granada: GEU.
Gairín, J. (1987). Proyecto docente. Departamento de Pedagogía y Didáctica, Universidad Autónoma de Barcelona (Documento policopiado, pp. 678).

Hopkins, D. (1996). Estrategias para el desarrollo de los centros educativos. En A. Villa (Coord.), Dirección participativa y evaluación de centros (pp. 377-402). Bilbao, España: Universidad de Deusto.

Howey, K. (1985). Six major functions of staff development: Ad expanded imperative [Seis funciones principales de desarrollo de personal: Un imperativo ampliado]. Journal of Teacher Education, 1(36), 58-64.

López, J. y Sánchez, M. (2004). La cultura institucional. En J. M. Moreno Olmedilla (Coord.), Organización y gestión de centros educativos. Madrid: UNED.

Lorenzo, M. (1994). Organización Escolar. La construcción de la escuela como ecosistema. Madrid: Ediciones Pedagógicas.

Martín, J. M. (2003). Las escuelas como organizaciones saludables. En Lorenzo, M. y otros (Coords.), Organización Escolar diferencial. Modelos y estrategias (pp. 13-24). Granada: GEU.

Medina, A. (1996). La cultura del centro educativo. En Lorenzo Delgado, M. (Coord.), La organización y gestión del centro educativo. Análisis de casos prácticos (pp. 299-323). Madrid: Universitas.

Medina, A. y Cardona, J. (1996). La construcción de unidades integradas: Una propuesta para alumnos/as con dificultades de aprendizaje. En Lorenzo, M. y Bolívar, A. (Coords.), Trabajar 
en los márgenes. Asesoramiento, formación e innovación en contextos educativos problemáticos (pp. 143-166). Granada: ICE de la Universidad.

Martín-Moreno, Q. (1996). La Organización de Centros Educativos en una perspectiva de cambio. Madrid: Sanz y Torres.

Nevis, E.C., Dibella, A. J. y Gould, J. M. (1995). Understanding organizations as learning systems [Entendiendo las organizaciones como sistemas de aprendizaje]. Sloan Management Review, 36(2), 73-85.

Santos, M.A. (1993). Estrategias para la evaluación interna de centros educativos. Madrid: MEC, Subdirección General de Formación del Profesorado.

Senge, P. (1992). La quinta disciplina. El arte y la práctica de la organización abierta al aprendizaje. Barcelona: Granica. 